This is the author's accepted manuscript. The final published version of this work (the version of record) is published by SAGE in Sage research methods available at:

DOI: http://dx.doi.org/10.4135/9781473989122. This work is made available in accordance with the publisher's policies. Please refer to any applicable terms of use of the publisher.

\title{
Class of 2010: a qualitative longitudinal study of an English secondary school that became an academy
}

Rowena Passy; Tanya Ovenden-Hope

\section{Keywords}

Longitudinal qualitative research; ethics; managing educational change

\begin{abstract}
This project was a qualitative longitudinal research (QLR) study into cultural and structural change in an English secondary school. The schools' consistent underperformance led to its conversion to 'academy' status as part of a development under the Labour administration of 1997-2010. The Labour policy for academisation was designed first, to challenge the culture of underachievement in areas of high deprivation and secondly, to encourage schools to play a part in local community regeneration. Our research began at the point of conversion to academy status, and we have subsequently visited the school each year to interview senior leadership, teaching staff and a sample of pupils to investigate their views on continuity and change within the institution and the impact on their own roles. Wider context for the research has been provided by annual publicly-available performance data and academy documentation.

In this case-study report we consider research design and some methodological issues that we encountered during the data collection. We show that establishing a relationship based on trust is a particularly important ingredient between researchers and participants in QLR if the project is to yield rich and interesting data.
\end{abstract}




\section{Learning Outcomes}

By the end of this case study the reader should be able to:

- Report the strengths and weaknesses of qualitative longitudinal research

- Identify some of the methodological issues in conducting longitudinal qualitative research

- State the main reasons why generating trust between researchers and participants in qualitative longitudinal research is important.

\section{Case Study}

\section{Project Overview and Context}

The context for this project was the UK Labour government's (1997-2010) policy to tackle persistent underperformance in the most challenging urban secondary school settings. The aim the policy was to create autonomous 'academies' that were independent of the local authority (local educational administration), that were managed by a team of independent cosponsors, and had the freedom to generate the kind of entrepreneurial leadership that was more usually associated with private sector business. The belief was that these structural changes would lead to substantial improvement in educational standards through a change in school culture in those areas. In practice, however, autonomy was relatively limited; while these new academies had the freedom to educate students in the way that they believed was most appropriate, they still had to comply with national policy demands in terms of student attainment in external examinations and inspection requirements.

Our case-study academy was located in a coastal area with high levels of socio-economic deprivation. At the time of conversion to academy status the school had the lowest examination results in the local authority which, when combined with strong competition from other high-performing schools (including grammar schools) and a local demographic trend of decreasing numbers of potential secondary students, meant that it was often the school of last resort. Students arrived with low levels of attainment by national benchmarks and had few local employment prospects on leaving school; briefly put, the academy suffered from a poor reputation in a community that had high levels of poverty and unemployment, and that seemingly placed little value on education. Nonetheless an experienced new Principal, together with support from the co-sponsors of a university, a further education college and the local authority, was judged to have the necessary expertise and capacity to turn the school's performance around. One of us (Tanya) was a Director of the new academy; she introduced the idea of the research to the leadership, who thought it could be provide a useful function for all involved - and so our qualitative longitudinal research (QLR) began in 2010 when the school became an academy.

\section{Why qualitative longitudinal research?}


Interest in qualitative longitudinal research (QLR) has increased over the last decade, and is linked to the idea of exploring social change over time. There are different types of QLR, with researchers such as Thomson and Macleod (2015, p.244) suggesting that it is 'increasingly understood as a sensibility and orientation rather than a specific research design'; the open-ended nature of the research (which can be undertaken for any length of time from months to decades), the different types of methods that can be used, and the flexibility needed to respond to issues that arise during the data collection period mean that this type of study tends to be developed on a case-by-case basis. As we discuss in the next section, our study involved repeated annual research visits to the academy in which we conducted interviews with academy leader(s), teachers and students. We think of the result as a photograph album that tells the story of this institution's life at particular moments over time.

The benefits of QLR are first, that it allows researchers to explore context, processes and their effects over time at the individual level. In a research project such as ours, in which the academy was expected to show a rapid improvement in students' examination results and in which organisational restructuring played a critical part in any projected improvement, the details of individual experiences gave us insight into the varied staff responses to an environment of rapid change; over time we could see differing patterns of response as some staff embraced / accommodated new developments, others chose to engage little and yet others chose to leave. Students, too, had issues that they raised during the course of our interviews; one example involved a rapid change in uniform requirements that raised costs which, in an area of high deprivation, students felt was unfair. As researchers, we wanted to ensure that all participants had a voice in all of our research reports, and feedback to the academy leadership on both staff and student views was a significant part of the project's integrity.

Secondly, the same researchers generally interview the same participants, enabling interviewers to question interviewees about issues they had raised and/or concerns expressed in the previous round. In our research we interviewed the same academy leaders and students but different teachers each year; this enabled a consistency of views from those leading the academy and from those who were experiencing the institution as learners, but encouraged a wide range of differing views from those who were responsible for carrying out the leadership's vision and teaching the students. This enabled us to have a wider and deeper understanding of the academy than if we had interviewed all the same research participants each year.

Thirdly, QLR enables researchers to link the macro processes (for school leaders, the policy context, for example; for teachers and students, the institutional context) to how change feels 'on the ground', making the research potentially useful to a range of different people. A particularly interesting aspect of our research, and one that we could not have foreseen, was that fundamental changes within the institution coincided with far-reaching changes within English secondary education; two different government administrations and a series of policy 
directives placed new pressures on the academy at a time in which leaders were already implementing extensive changes aimed at the primary measure of school accountability student performance in formal examinations. This makes the study particularly valuable as a means of illustrating policy impacts at a challenging time in English secondary education.

Equally, as Farrall (2006) has commented, QLR has weaknesses as a research approach. The first is that it is highly time- and resource-intensive, requiring considerable investment in researchers' time to set up, prepare and conduct interviews, in interview transcription and in subsequent data analysis, which becomes increasingly complex as new waves of data collection are completed. This means that QLR tends to be expensive, and usually focuses on a relatively small group of participants. We were fortunate in terms of funding; our time, as university employees whose job descriptions included engaging in research, was already covered, and we organised and undertook the research ourselves. This meant that additional expenses of transcription and occasional research assistance were kept to a minimum, and had the effect of keeping us close to the research process itself; regular contact with the school leaders to set up the visits, mediate any issues related to the research and send the reports meant that we built long-term relationships with different people within the institution. In turn, they sent us invitations to significant events that involved 'our' students, such as an end-of-year performance, which helped to strengthen those ties. We discuss the potential difficulties and implications of developing this type of relationship further in Section 4.

Secondly change takes time and, although some studies may yield important findings fairly quickly, it may take several years before the nature, extent and impact of any change becomes evident. In our research, there was both rapid and longer-term change; initial measures were taken to improve the immediate levels of student performance, but there was also the longer-term aim of shifting the school culture from one of low aspirations towards one of engaging students with their learning, fostering high expectations for both teachers and students, and achieving consistently higher levels of attainment. Collarbone argues that the development of an 'autonomy culture' takes time, and she advocates an 'evolutionary approach' (Collarbone, 2012, pp.2-3) which, broadly, was the approach taken within this academy. Thus new structures and approaches to teaching and learning were brought in, modified and, in some cases (such as 100-minute lessons), abolished in response to new policy demands; many of these developments were discussed at the three levels of leadership, staff and student, enabling us to monitor and interpret both the short- and longer-term changes over time. Gradually the interview data allowed us to begin to answer the question: '(How) has the school culture changed?'

Thirdly, there is always attrition in a sample of interviewees - which means that the number of interviewees should initially be larger than the desired sample number, and which again adds to the investment required. Finally, interviewees may suffer what has been termed 'question fatigue'; if the same questions are used repeatedly, there is a danger that the interview can fail to engage the participant in meaningful discussion, resulting in a series of 
answers that are essentially 'going through the motions' or withdrawal from the study. We discuss these issues further in Section 4.

In the next section we describe our research design, and follow this with reflection on the issues that we encountered during the course of data collection and analysis.

\section{Research design}

We felt that the new structure of the school, together with the wide-reaching ambitions of the academy leaders that - in line with the government policy - included raising academic performance and playing a part in local community regeneration through developing business links and leisure facilities, offered a unique opportunity to document and explore the impact of the (relatively) new policy. How would the new academy develop? What challenges would the new leaders face? What would be the impact of changes within the academy on staff and students? Would the academy achieve its early aims? How much would the sponsors be involved in the transition to academy status and the school that emerged from this?

Academy leaders and sponsors agreed to a seven-year longitudinal study which would include the secondary educational experience of the Year 7 cohort (the youngest students who entered the academy at the time of conversion, whom we named the Class of 2010), who were legally obliged to attend school until the age of 16, and then to attend either school or a training establishment from the ages of 16-18. The hope was that the majority of students would remain at the academy for their 16-18 education. The aims of the study were to:

- explore the senior leadership aims and priorities, and how these might change and develop over time.

- investigate teachers' perceptions of the teaching and learning methods employed by the academy.

- monitor the academic progress of the student cohort that entered the academy in Year 7 (the first year of secondary school) in the year of conversion to academy status.

- understand the educational journey of a sample of 15 Year 7 students.

The study methods were an annual school visit that included:

- interviewing the principal and/or a senior leader to understand strategic priorities, challenges and successes

- interviewing a sample of four teachers to gain a range of different views on the impact of the measures undertaken each year

- interviewing the sample of 15 Year 7 students to investigate the impact of different policies and procedures on their school experience.

- collecting the anonymised cohort data on levels of progress and attainment.

In addition we studied the academy's publicly-available data to provide context for the annual research visit, and examined academy documentation such as the Improvement Plan, 
organisational structure, and relevant policies. Our immediate responsibility to the academy was to provide an annual report on the research findings.

\section{Research in practice}

As academic researchers, our job is to provide an independent view of 'what is going on' within our chosen research areas. That does not mean that we believe in objectivity in social science; our view is that there is no one version of the truth ready to be harvested as if it were wheat or oats, but that there are different versions depending on context, personal beliefs, values and attitudes. Our methods were therefore designed to give a range of perspectives on developments within the academy, triangulated further through reference to its performance measures and documentation, so that we could feel confident that we were able to offer a careful analysis of academy developments, challenges and successes. However straightforward this approach might seem, it nonetheless produced two notable challenges in data collection as the project progressed.

The first was our developing relationship with the academy, linked in the first place to the selection of four teachers to interview each year. This is always a difficult position for a researcher; generally s/he knows neither the different teachers (and in a large secondary school there can be well over 100) nor their timetables, and the research visit generally has to be completed in a day to minimise disruption to the school. The researcher is also aware that permission for the research can be withdrawn from the school at any time, and generally tries to make the process as uncomplicated as possible. For all these reasons we asked the school leaders to select the teachers to be interviewed, with the only criterion being that they should be involved in teaching the cohort of students that we were following. This was a political decision that depended partly on the school leaders' attitude to the research; would they allow us to interview dissident teachers at a time of change and draw on these views to support the process? Or would they only allow those who generally supported leadership decisions? Our promise of confidentiality to the teachers (that is, no comments would be attributed to individuals in the reports) provided some identity protection, but leadership nonetheless knew that the views could only come from a limited number of people. How would teachers respond?

In practice we found that all this depended, as with the students, on the relationship of trust that we built up with academy. In Section 2 we outlined how our relationship grew with individuals at the academy, and how we were invited to participate in academy events that involved 'our' students. One the one hand, this was highly positive. In the first two years of the research we found that teachers tended to have generally favourable views on leadership decisions, and decided that this may have been partly because the majority had been employed by the new Principal (and therefore supported her approach), and partly because the leadership were wary of allowing potentially rebellious teachers to speak their mind to a member of the governing body. In the third year of the research, however, we asked the leadership to ensure that we had a variety of teacher views on the academy's development, explaining that the research would be more helpful to them if that were the case. By this time 
Tanya was established as an effective director, we had created positive working relationships with different individuals in the academy and had submitted two reports, using the research data sensitively to provide accounts that raised participants' issues (students' and teachers') as well as outline successes and challenges. As a result, the leadership seemed to feel that we could be trusted; subsequent teacher interviews provided a more realistic balance between support and critique and yielded richer data that, in turn, enabled a more detailed and nuanced report. As we had suggested, the leadership found this was more useful to the academy, and Tanya was invited to discuss subsequent reports at governors' meetings.

One the other hand, developing a closer relationship with the academy as a whole could be seen to encourage us to lose our critical distance from the research; that we could become too involved with the academy to provide an independent view of processes and events. Mercer (2007), however, argues that the researcher's position is often on a continuum of insideroutsider, that this position fluctuates depending on a range of diverse characteristics including gender, type of employment and - even - the topic of conversation, and that all research presents practical problems, regardless of individual researchers' positions. Our experience was that the combination of Tanya's role as director and our ongoing relationship with the academy brought both advantages and disadvantages, most of which are unquantifiable. Access to the research site was possibly initially easier for us through Tanya's position, although it was equally possible that another Principal would have been suspicious of the research at such a critical time; knowing that one of the interviewers was a governor could have caused teachers to modify their responses to what they thought she wanted to hear, or it could have encouraged them to talk more freely because they felt she had influence over the academy leadership. Tanya had a degree of inside knowledge about academy policies, and this enabled us to question participants about the detail of, for example, the way behaviour policies worked in practice; equally this 'heightened familiarity' (Mercer, 2007, p.11) may have meant that we missed obvious questions or failed to challenge assumptions. Thinking about these issues made us doubly careful about framing the teacher and student interview questions so they were sufficiently open that all participants could answer in any way they chose; that follow-up questions were neutral in approach; and that we avoided references to Tanya's position as director. We critically evaluated each set of interviews before drawing up the next interview schedules to ensure these principles were followed each year. Analysis of the different types of data was undertaken thematically with critical support from a Research Assistant to ensure a balanced approach. In short, we undertook regular checks and balances to make certain that the research represented a careful analysis of 'what was going on' within the academy.

The second was student attrition. While we expected this, we knew that student participants needed to be engaged with the research and feel that it had some purpose for them to want to continue; all volunteered for the research and had the right to withdraw at any point. Our approach was to tell the students that their views would be fed back to school leaders, and to show them how this had been done by writing an annual student report that outlined the points they had raised. We tried to make the interview process enjoyable by ensuring that we 
took a personal interest in each student and that, as far as possible, the same researcher interviewed the same students in order to build up a relationship. We also took in a tin of sweets, to be shared among the students! Over time, the students divided into roughly two groups; those that became involved with the research and were increasingly willing to share their views, and those who lost interest. Altogether seven of the fifteen students withdrew from the study, most of whom were male. Although the sample at Year 5 of the study was small and had a high proportion of females, we were fortunate that it still represented a broad range of abilities and interests, with these students offering highly valuable thoughts and comments that ranged from highly critical to high praise.

Student attrition highlighted the issue of the semi-structured interview schedule. We wanted to repeat this during the years of study so that we could monitor attitude change towards particular areas of school organisation such as classroom management, extra-curricular activities and the role of trips in learning. We found 'question fatigue' became less of a problem as the students who were interested and engaged in the research grew older; these students became increasingly willing to discuss their views as the research progressed and our relationships deepened. Those who were less interested tended to become monosyllabic, however, suggesting that a different approach would have been helpful to engage their interest - a point reinforced by the relatively high drop-out rate of male participants. We relied on students' memory to tell us the high and low points of their year, for instance, which those who did not particularly enjoy school or perhaps were experiencing personal problems found difficult. In retrospect, we can see it would have been helpful to find out information relevant to the cohort (such as a trip to an adventure learning facility, or the introduction of a different behaviour policy, for instance) before each visit and used these as something specific for interviewees to comment on. This approach may have encouraged the more reluctant to open a conversation rather than merely respond to questions, and thus to engage with the research more readily.

\section{Conclusion}

QLR has enable us to establish a long-term relationship with an academy in a way that has generated rich, detailed and valuable data on efforts to transform a school from low- to highperforming at a challenging time in English education. The key was to develop trust between project and participants at all levels of the research; to demonstrate that we were genuinely interested in what happened, that we were committed to the research, that we understood the issues faced, and that we used data sensitively to raise interesting and relevant points to support this process of change. In discussing some of the practical issues that we encountered, we hope that we have shown some useful and practical ways in which to engage institutions and students with long-term research. 


\section{Exercises and Discussion Questions}

[4 - 8 questions on your case (topic or method) suitable for classroom use]

1. We describe our research as 'a photograph album that tells the story of one institution's life at particular moments over time'. How else might it be described? What implications does this visualisation have for any research process?

2. Think of ways in which you would engage secondary-aged students with long-term research.

3. Think of a topic that might be suitable for qualitative longitudinal research. How would you design the research? Questions you would need to consider would be how you would access the site(s), the duration of the study, who and what to include, the regularity of data collection, and the reporting mechanisms.

4. In what circumstances would you use quantitative longitudinal research rather than qualitative longitudinal research?

\section{Further Readings}

Holland, J., Thomson, R. \& Henderson, S. (2006) Qualitative Longitudinal Research: A Discussion Paper, https://www.lsbu.ac.uk/_data/assets/pdf_file/0019/9370/qualitativelongitudinal-research-families-working-paper.pdf.

\section{Web Resources}

None

\section{References}

Collarbone, P. (2012) Leading change, changing leadership. Occasional paper 126, Centre for Strategic Education. Retrieved from http://www.cse.edu.au/zfiles/CSE-PatCollarbone.pdf.

Farrall, S. (2006) What is Qualitative Longitudinal Research? Papers in Social Research Methods, Qualitative Series no 11. Retrieved from http://www.lse.ac.uk/methodology/pdf/QualPapers/Stephen-FarrallQual\%20Longitudinal\%20Res.pdf.

Mercer, J. (2007) The Challenges of Insider Research in Educational Institutions: Wielding a 
double-edged sword and resolving delicate dilemmas, Oxford Review of Education, 33(1), 117, DOI: 10.1080/03054980601094651.

Thomson, R. \& Macleod, J. (2015) New frontiers in qualitative longitudinal research: an agenda for research, International Journal of Social Research Methodology, 18:3, 243-250, DOI: $10.1080 / 13645579.2015 .1017900$. 